Pacific Journal of Mathematic 


\section{ABELIAN GROUPS CHARACTERIZED BY THEIR INDEPENDENT SUBSETS}

\section{Wu-Chung Hsiang}

1. Introduction. Recently, the theory of abelian groups has become an active field. This note is devoted to it. One of the important theorems, called " the theorem on the subgroups of a free abelian group $U_{n}$ of finite rank $n[2, \mathrm{p}$. 145] will be studied here. From now on the term "group" will be used instead of "abelian group", for simplicity. Since the notations, the definitions, and the terminologies vary for different authors, we refer to Kurosh [2] and Kaplansky [1] as standards. For example, for the definitions of the height of an element $x$ in a primary group $G$ (denoted by $h_{G}(x)$ ), of the lowest layer of a primary group we refer to [2], and the definitions, of $Z(n)$, of $Z\left(p^{\infty}\right)$ we refer to [1]. Moreover, the subgroups spanned by the subset $\left\{u_{\alpha}\right\}$ of a given group $G$ is denoted by $\left(\left\{u_{\alpha}\right\}\right)$, and in a primary group we write $o(x)=n$ if the order of the element $x$ is equal to $p^{n}$.

For convenience, the following terminology is adopted.

I. A group $G$ has property (A), if for any non-zero element $x$ of $G$ there exists a cyclic direct summand of $G$ containing $x$.

II. A group $G$ has property (B), if $G$ is a direct sum of cyclic groups, and for any subgroup $H$ of $G$ there exists a basis $\left\{h_{\alpha}\right\}$ of $H$ and a basis $\left\{g_{\beta}\right\}$ of $G$ such that for any $h_{\alpha} \in\left\{h_{\alpha}\right\}$ we can find a $g_{\alpha} \in\left\{g_{\beta}\right\}$ with the property $h_{\alpha} \in\left(g_{\alpha}\right)$.

III. A group $G$ has property (C), if for any independent subset $\left\{h_{x}\right\}$ of $G$ there exists another independent subset $\left\{g_{\alpha}\right\}$ of $G$ such that $h_{\alpha} \in\left(g_{\alpha}\right)$ and $\left(\left\{g_{\alpha}\right\}\right)$ is a direct summand of $G$.

The purpose of this paper is to give an analysis of these classes of groups. In particular, we show that a free group $U_{n}$ of finite rank $n \geqq 2$ has properties (A) and (B) but not (C).

General Lemma. A torsion group $G$ has property (A), (B) or (C) respectively, if and only if each of its primary components has property (A), (B) or (C) respectively.

Proof. We decompose $G$ into its primary components,

$$
G=\sum_{p} \oplus G_{p} .
$$

We prove that $G$ has property (C) if and only if each $G_{p}$ has property (C). If for an independent subset $\left\{x_{a}^{(p)}\right\} \subset G_{p}$, we can find an independent subset $\left\{g_{\alpha}\right\} \subset G$ such that $x_{\alpha}^{(p)} \in\left(g_{\alpha}\right)$ for all $\alpha$, and the subgroup

Received September 17, 1957. In revised form, May 1, 1958. 


$$
\sum_{\alpha} \oplus\left(g_{\alpha}\right)
$$

is a direct summand of $G$, then we can find a subset $\left\{g_{\alpha}^{(p)}\right\} \subset G_{p}$ such that $x_{\alpha}^{(p)} \in\left(g_{\alpha}^{(p)}\right)$ and $\sum_{\alpha} \oplus\left(g_{\alpha}^{(p)}\right)$ is a direct summand of $G_{p}$. To find the desired subset $\left\{g_{a}^{(p)}\right\} \subset G_{p}$, we need only consider the $p$-components of $g_{\alpha}$ 's, since $x_{\alpha}^{(p)} \in\left(g_{\alpha}\right)$ implies that $x_{\alpha}^{(p)}$ is contained in the $p$-component of $\left(g_{\alpha}\right)$ which is a cyclic subgroup of $G_{p}$ generated by the $p$-component of $g_{\alpha}$.

Conversely, if for every $p$-component $\left\{x_{a}^{(p)}\right\} \subset G_{p}$ of an independent subset $\left\{x_{\alpha}\right\} \subset G$, we can find an independent subset $\left\{g_{\alpha}^{(p)}\right\}$ such that $x_{\alpha}^{(p)} \in\left(g_{\alpha}^{(p)}\right)$ for all $\alpha$ 's and the subgroup $\sum_{\alpha} \oplus\left(g_{\alpha}^{(p)}\right)$ is a direct summand of $G_{p}$, then there exists an independent subset $\left\{g_{\alpha}\right\} \subset G$ such that $x_{\alpha} \in\left(g_{\alpha}\right)$ for all $\alpha$ 's and the subgroup $\sum_{\alpha} \oplus\left(g_{\alpha}\right)$ is a direct summand of $G$. Since every $x_{a}$ has only a finite number of $p$-components different from zero, we can set $g_{a}^{(p)}=0$ when $x_{a}^{(p)}=0$. To find the desired subset $\left\{g_{\alpha}\right\} \subset G$, we need only set

$$
g_{\alpha}=\sum_{p} g_{\alpha}^{(p)} .
$$

The independence of $\left\{g_{\alpha}\right\}$ follows from the independence of $\left\{g_{\alpha}^{(p)}\right\}$ for all $p$, and $x_{\alpha} \in\left(g_{\alpha}\right)$ follows from

$$
\left(x_{\alpha}\right)=\sum_{p} \oplus\left(x_{\alpha}^{(p)}\right) \subset \sum_{p} \bigoplus\left(g_{\alpha}^{(p)}\right)=\left(g_{\alpha}\right) .
$$

The other two parts of the lemma are proved in an analagous manner.

2. Groups with property (A). To discuss groups with property (A), we need several lemmas.

LEMMA I. If $G$ is a primary cyclic group of the type $Z\left(p^{n}\right)$, and $x$ is a non-zero element of $G$, then

$$
o(x)+h_{G}(x)=n \text {. }
$$

This is clear.

LEMMA II. If $G$ is a primary group of the type

$$
Z\left(p^{h_{1}}\right) \oplus Z\left(p^{h_{2}}\right) \oplus H
$$

where $3 \leqq h_{1}+2 \leqq h_{2}$, then it is not a group with property (A).

Proof. Let $u_{1}, u_{2}$ be the generators of $Z\left(p^{h_{1}}\right), Z\left(p^{h_{2}}\right)$ respectively. Therefore,

$$
o\left(u_{2}\right)=h_{2} \geqq h_{1}+2=o\left(u_{1}\right)+2 .
$$

To prove Lemma II, it is sufficient to show that there does not exist 
any cyclic direct summand of $G$ containing $p^{h_{1}-1} u_{1}+p^{h_{1}} u_{2}$. Suppose the contrary. Then, we can find a cyclic direct summand $V=(v)$ containing $p^{h_{1}-1} u_{1}+p^{h_{1}} u_{2}$. Since $h_{2}-2 \geqq h_{1}$, it is clear that the lowest layers of $V$ and of the cyclic group $\left(p^{h_{1}-1} u_{1}+p^{h_{1}} u_{2}\right)$ are both equal to $\left(p^{h_{2}-1} u_{2}\right)$, and $o\left(p^{h_{1}-1} u_{1}+p^{h_{1}} u_{2}\right)=h_{2}-h_{1}$. Let $o(v)=k$. Since $(v)$ and $\left(u_{2}\right)$ are direct summands of $G$,

$$
h_{G}\left(p^{k-1} v\right)=h_{v}\left(p^{k-1} v\right)=k-1
$$

and

$$
h_{G}\left(p^{h_{2}-1} u_{2}\right)=h_{V}\left(p^{h_{2}-1} u_{2}\right)=h_{2}-1 .
$$

But since the lowest layer of $(v)$ is $\left(p^{k-1} v\right)=\left(p^{h_{2}-1} u_{2}\right)$, we have $k-1=$ $h_{2}-1$, and $o(v)=k=h_{2}$. Therefore, it follows from Lemma I that

$$
h_{V}\left(p^{h_{1}-1} u_{1}+p^{h_{1}} u_{2}\right)=h_{2}-\left(h_{2}-h_{1}\right)=h_{1} .
$$

On the other hand, we have

$$
h_{G}\left(p^{h_{1}-1} u_{1}+p^{h_{1}} u_{2}\right)=\min \left(h_{1}-1, h_{1}\right)=h_{1}-1 .
$$

This is a contradiction since $V=(v)$, being a direct summand, is a pure subgroup of $G$, so that for $x \in V, h_{V}(x)=h_{G}(x)$.

Lemma III. A primary group $G$ has property (A) if and only if it is of the type

$$
\sum_{\rho} \oplus Z_{\rho}\left(p^{h}\right) \oplus \sum_{\lambda} \oplus Z_{\lambda}\left(p^{h+1}\right) .
$$

Proof. First, $G$ must be a reduced group. If this is not the case, we can find a non-zero $x$ of $G$ which is of infinite height in $G$. It is clear that $x$ cannot be contained in a cyclic direct summand $(g)$ of $G$. Second, $G$ must be of bounded order, therefore $G$ is a direct sum of cyclic groups. Otherwise, by [1, Theorem 9] there exists a direct summand $\left(u_{1}\right) \oplus\left(u_{2}\right)$ of $G$ of the type

$$
Z\left(p^{h_{1}}\right) \oplus Z\left(p^{k_{2}}\right), \quad 3 \leqq h_{1}+2 \leqq h_{2} .
$$

Then, by Lemma II, $G$ is not a group with property (A). Moreover, the bounded group $G$ must be of the type

$$
\sum_{\rho} \oplus Z_{\rho}\left(p^{h}\right) \oplus \sum_{\lambda} \oplus Z_{\lambda}\left(p^{h+1}\right) .
$$

Conversely, if $G$ is of the type

$$
\sum_{\rho} \oplus Z_{\rho}\left(p^{h}\right) \oplus \sum_{\lambda} \oplus Z_{\lambda}\left(p^{h+1}\right)
$$

we claim that it is a group with property (A). Let $\left\{u_{\rho}\right\}$ and $\left\{u_{\lambda}\right\}$ be 
the generators of the cyclic subgroups of the type $Z_{\rho}\left(p^{h}\right)$ and $Z_{\lambda}\left(p^{h+1}\right)$ respectively. Then, for any non-zero $x \in G$, we can express it as

$$
\sum_{1}^{s} a_{i} p^{l_{i}} u_{\rho_{i}}+\sum_{s+1}^{t} b_{j} p^{l} j u_{\lambda_{j}}
$$

such that $\left(a_{i}, p\right)=\left(b_{j}, p\right)=1$ and $0 \leqq l_{i}<h, 0 \leqq l_{j} \leqq h$.

It is obvious that

$$
\begin{aligned}
h_{G}(x) & =\min \left(l_{1}, \cdots, l_{s}, l_{s+1}, \cdots, l_{t}\right) \\
o(x) & =\max \left(h-l_{1}, \cdots, h-l_{s}, h+1-l_{s+1}, \cdots, h+1-l_{t}\right) .
\end{aligned}
$$

If $o(x)$ is equal to a certain $h-l_{i}(1 \leqq i \leqq s)$, that is $h-l_{1}$, then the generator $\tilde{x}$ of the lowest layer of $(x)$ is of height $h-1$ in $G$. Since

$$
\begin{array}{lr}
h-l_{1} \geqq h-l_{i} & (1 \leqq i \leqq s) \\
h-l_{1} \geqq h+1-l_{j} & (s+1 \leqq j \leqq t),
\end{array}
$$

we have

$$
\begin{array}{rr}
l_{1} \leqq l_{i} \\
l_{1}<l_{j} & (1 \leqq i \leqq s) \\
& (s+1 \leqq j \leqq t) .
\end{array}
$$

Hence, the cyclic subgroup

$$
\left(\sum_{1}^{s} a_{i} p^{l_{i}-l_{1}} u_{\rho_{i}}+\sum_{s+1}^{t} b_{j} p^{l_{j}-l_{1}} u_{\lambda_{j}}\right)
$$

is pure, and by [1, Theorem 7] is a direct summand, which contains the element $x$. The cyclic subgroup is pure by [1, Lemma 7].

If $o(x)$ is equal to a certain $h+1-l_{f}(s+1 \leqq j \leqq t)$, that is $h+1-l_{s+1}$, but not equal to any $h-l_{i}(1 \leqq i \leqq s)$, then the generator $\tilde{x}$ of the lowest layer of $(x)$ is of height $h$ in $G$. We have

$$
\begin{array}{lr}
l_{i}>l_{s+1}-1 & (1 \leqq i \leqq s) \\
l_{j} \geqq l_{s+1} & (s+1 \leqq j \leqq t) .
\end{array}
$$

Consequently, by the same argument as we used above,

$$
\left(\sum_{1}^{s} a_{i} p^{l_{i}-1} s+1 u_{\rho_{i}}+\sum_{s+1}^{t} b_{j} p^{l_{j}-l_{s+1}} u_{\lambda_{j}}\right)
$$

is the desired direct summand containing $x$. This proves our lemma.

Theorem $\mathrm{A}_{1}$. A group $G$ with property (A) cannot be mixed, i.e., it is either torsion or torsion-free. Moreover, a torsion group $G$ has property (A) if and only if $G$ is of the type

$$
\sum_{p} \oplus\left(\sum_{\alpha_{p}} \oplus Z_{\alpha_{p}}\left(p^{h} p\right) \oplus \sum_{\beta_{p}} \oplus Z_{\beta_{p}}\left(p^{h} p^{+1}\right)\right) \text {. }
$$


Proof. If $G$ is a torsion group, the theorem follows from the General lemma, and Lemma III. Now, we are going to prove that $G$ cannot be mixed. If $G$ has property (A), then the torsion subgroup $T(G)$ of $G$ must be of the type

$$
\sum_{p} \oplus\left(\sum_{\alpha_{p}} \oplus Z_{\alpha_{p}}\left(p^{h_{p}}\right) \oplus \sum_{\beta_{p}} \oplus Z_{\beta_{p}}\left(p^{h_{p}+1}\right)\right) .
$$

If this is not the case, $T(G)$ does not have property (A), and there exists a non-zero element $x \in T(G)$ which is not contained in any cyclic direct summand $(g)$ of $T(G)$, and therefore, it cannot be contained in a cyclic direct summand of $G$. We shall prove that if $T(G) \neq 0$, then $G=T(G)$. Suppose the contrary. There exists a non-zero $x \in G$, such that we can find an infinite cyclic direct summand $\left(u_{1}\right)$ of $G$ containing $x$. Select a non-zero primary component $T_{p_{1}}(G)$ of $G$, which is of the type

$$
\sum_{\lambda_{p_{1}}} \oplus Z_{\lambda_{p_{1}}}\left(p_{1}{ }^{{ }_{p_{1}}}\right) \oplus \sum_{\mu_{q_{1}}} \oplus Z_{\mu_{p_{1}}}\left(p^{{ }^{{ }_{p_{1}}}+1}\right)
$$

We have

$$
G=\left(u_{1}\right) \oplus T_{p_{1}}(G) \oplus G^{\prime},
$$

by [ 1 Theorem 7]. Consider the element $p_{1}^{s} u_{1}+a_{p_{1}}$, where $s>h_{p_{1}}+1 \geqq 1$ and $h_{p_{1}}(G)\left(a_{p_{1}}\right)=0$. Since $G$ has property $(\mathrm{A})$, there exists a cyclic direct summand $(g)$ containing $p_{1}^{s} u_{1}+a_{p_{1}}$. We have

$$
g=l_{1} u_{1}+b_{p_{1}}+g^{\prime}, \text { where } g^{\prime} \in G^{\prime}, b_{p_{1}} \in T_{p_{1}}(G) .
$$

By the hypothesis, there exists an integer $l$ such that

$$
l g=l l_{1} u_{1}+l b_{p 1}+l g^{\prime}=p_{1}^{s} u_{1}+a_{p_{1}} .
$$

Consequently, we have

$$
l=p_{1}^{c}, l_{1}=p_{1}^{a} \text {, and } g^{\prime}=0, \text { where } c+d=s .
$$

Thus

$$
g=b_{p_{1}}+p_{1}^{d} u_{1} \text {, and } a_{p_{1}}=p_{1}^{c} b_{p_{1}} .
$$

Since $h_{\left.T_{p_{1}}{ }^{G}\right)}\left(a_{p_{1}}\right)=0$, we must have also $c=0$, that is $l=1$ and $g=$ $p_{1}^{s} u_{1}+a_{p_{1}}$. Hence $\left(p_{1}^{s} u_{1}+a_{p_{1}}\right)$ is a direct summand of $G$. Since

$$
\left(p_{1}^{s} u_{1}+a_{p_{1}}\right) \subset T_{p_{1}}(G) \oplus\left(u_{1}\right),
$$

we have that $\left(p_{1}^{s} u_{1}+a_{p_{1}}\right)$ is also a cyclic direct summand of $T_{p_{1}}(G) \oplus\left(u_{1}\right)$. It is clear that

$$
T_{p_{1}}(G) \oplus\left(u_{1}\right)=T_{p_{1}}(G) \oplus\left(p_{1}^{s} u_{1}+a_{p_{1}}\right)
$$


Then

$$
u_{1}=f\left(p_{1}^{s} u_{1}+a_{p_{1}}\right)+b_{p_{1}}=f p_{1}^{s} u_{1}+f a_{p_{1}}+b_{p_{1}}
$$

for a non-zero integer $f$, and an element $b_{p_{1}} \in T_{p_{1}}(G)$. It follows that $u_{1}=f p_{1}^{s} u_{1}$, which contradicts $s>1$. Therefore the proof is complete.

THEOREM $\mathrm{A}_{2}$. A free group $G$ has property (A).

Proof. Since $G=\sum \nu \oplus\left(u_{\nu}\right)$, each $x \neq 0$ in $G$ can be written $x=a_{1} u_{\nu_{1}}+a_{2} u_{\nu_{2}}+\cdots+a_{n} u_{\nu_{n}}$. Hence

$$
G=\sum_{1}^{n} \oplus\left(u_{\nu_{i}}\right) \bigoplus_{\nu \neq \nu_{1}, \cdots \nu_{n}} \bigoplus_{\nu}\left(u_{\nu}\right) \text {, }
$$

and it follows from the theorem on the subgroups of a free group of rank $n\left[2\right.$, p. 145], that there exist a basis $v_{1}, v_{2}, \cdots, v_{n}$ of $\sum_{1}^{n} \oplus\left(u_{v_{i}}\right)$ such that $x \in\left(v_{1}\right)$. But $\left(v_{1}\right)$ is a direct summand of $G$, so that $G$ has property (A).

REMARK. ${ }^{1}$ A torsion-free group with property (A) need not be a free group. The counter-example is the unrestricted direct sum of infinitely many infinite cyclic groups. This group has property (A) and is not free [2, p. 216].

EXAMPLE. Let $G$ be the unrestricted direct sum of infinitely many infinite cyclic groups. Then $G$ has property (A).

Proof. Let $g \in C, g=\sum_{i=1}^{\infty} k_{i} a_{i}$, where $G$ is the unrestricted direct sum of infinite cyclic groups $\left(a_{i}\right)$. Let $m=\left(k_{i}, l=1,2, \cdots\right)$. Then g.c.d. there exists a finite subset of the $k_{i}$, say $k_{1}, k_{2}, \cdots, k_{n}$, such that g.c.d. $\left(k_{1}, k_{2}, \cdots, k_{n}\right)=m$. Then we have

$$
g=\sum_{i=1}^{\infty} k_{i} a_{i}=\sum_{i=1}^{\infty} m l_{i} a_{i}=m\left[\sum_{i=1}^{n} l_{i} a_{i}+\sum_{i=n+1}^{\infty} l_{i} a_{i}\right]
$$

where g.c.d. $\left(l_{1}, \cdots, l_{n}\right)=1$. Let

$$
g_{1}=\sum_{i=1}^{n} l_{i} a_{i}+\sum_{i=n+1}^{\infty} l_{i} a_{i}
$$

and

$$
H=\left\{h=\sum_{i=1}^{\infty} \alpha_{i} a_{i} \in G \mid \alpha_{i}=0, i=1,2, \cdots, n\right\} .
$$

Then $G / H$ is isomorphic to $\sum_{1}^{n} \oplus(a)$, a free group of rank $n$. Now,

$$
\left(H, g_{1}\right) / H=\left(\sum_{i=1}^{n} l_{i} a_{i}+H\right)
$$

1 This remark and the following example are due to R. A. Beaumont. 
is a cyclic subgroup of $G / H$. By the theorem on subgroups of a free group of rank $n$, there exists a basis $v_{1}, v_{2}, \cdots, v_{n}$ of $G / H$ such that

$$
\sum_{i=1}^{n} l_{i} a_{i}+H \in\left(v_{1}\right) \text {. }
$$

Thus

$$
\sum_{i=1}^{n} l_{i} a_{i}+H=e v_{1}=e \sum_{i=1}^{n} \beta_{i} a_{i}+H,
$$

so that $\left(e \beta_{i}-l_{i}\right) \alpha_{i}=0, i=1,2, \cdots, n$. Hence $l_{i}=e \beta_{i}, i=1,2, \cdots, n$ and

$$
\text { g.c.d. }\left(l_{1}, l_{2}, \cdots, l_{n}\right)=\text { g.c.d. }\left(e \beta_{1}, e \beta_{2}, \cdots, e \beta_{n}\right)=e=0 \text {. }
$$

We have proved $\sum_{i=1}^{n} l_{i} a_{i}+H=v_{1}$, so that

$$
G / H=\left(\sum_{i=1}^{n} l_{i} a_{i}+H\right) \oplus\left(v_{2}\right) \oplus \cdots \oplus\left(v_{n}\right)=\left(g_{1}+H\right) \oplus G^{\prime} \mid H .
$$

Then $G=\left(g_{1}\right) \oplus G^{\prime}$, and $g=m g_{1} \in\left(g_{1}\right)$. The problem of characterization of torsion-free groups with property (A) is still open.

3. Groups with property (B). We prove first that for primary groups, properties (A) and (B) are equivalent.

Lemma IV. A primary group $G$ has property (B) if and only if it is of the type

$$
\sum_{\lambda} \oplus Z_{\lambda}\left(p^{h}\right) \oplus \sum_{\mu} \oplus Z_{\mu}\left(p^{h+1}\right) .
$$

Proof. The necessary condition follows from Lemma III since property (B) implies property (A). Conversely, suppose that $G \cong M \oplus N$ where

$$
M \cong \sum_{\lambda} \oplus Z_{\lambda}\left(p^{h}\right) \text { and } N \cong \sum_{\mu} \oplus Z_{\mu}\left(p^{h+1}\right)
$$

Let $H$ be a subgroup of $G$. Then, ${ }^{2}(\bar{H} \cap \bar{M}) \subset \bar{H}$. Since both $\bar{H} \cap \bar{M}$ and $\bar{H}$ are of bounded height in $H$, by [1, Lemma 11], applied to the primary group $H$, we have a basis $\left\{h_{\alpha_{1}}\right\} \cup\left\{h_{\alpha_{2}}\right\}$ of $H$ such that

$$
\overline{\sum_{\alpha_{1}} \oplus\left(h_{\alpha_{1}}\right)}=(\bar{H} \cap \bar{M})
$$

and

$$
\overline{\sum_{\alpha_{1}} \oplus\left(h_{\alpha_{1}}\right)} \oplus \overline{\sum_{\alpha_{2}} \oplus\left(h_{\alpha_{2}}\right)}=\bar{H} .
$$

${ }^{2}$ We denote the lowest layer of a primary group $K$ by $\bar{K}$. Thus $\bar{K}=\{x \in K \mid p x=0\}$. 
By Lemma III, every $h_{\alpha}$ is contained in a cyclic direct summand $\left(g_{\alpha}\right)$ of $G$. We are going to prove that $\left\{g_{\alpha}\right\}=\left\{g_{\alpha_{1}}\right\} \cup\left\{g_{\alpha_{2}}\right\}$ is a pure independent subset of $G$. The independence of $\left\{g_{\alpha}\right\}$ follows from the independence of $\left\{h_{\alpha_{1}}\right\} \cup\left\{h_{\alpha_{2}}\right\}$. We need only prove that

$$
G_{1}=\sum_{\alpha} \oplus\left(g_{\alpha}\right)=\sum_{\alpha_{1}} \oplus\left(g_{\alpha_{1}}\right) \oplus \sum_{\alpha_{2}} \oplus\left(g_{\alpha_{2}}\right)
$$

is pure in $G$. It is sufficient to prove that every $g_{1} \in \bar{G}_{1}$ has the same height in $G_{1}$ as in $G$, by [1, Lemma 7]. The element $g_{1}(\neq 0) \in \bar{G}_{1}$ can be written as

$$
g_{1}=p^{h-1}\left[\sum_{1}^{s} l_{i} g_{\alpha_{i}}\right]+p^{h}\left[\sum_{s+1}^{t} l_{j} g_{\alpha_{2_{j}}}\right]
$$

where $\left(l_{i}, p\right)=\left(l_{j}, p\right)=1$.

If the first sum does not occur in the above expression for $g_{1}, h_{G_{1}}\left(g_{1}\right)=h$, which is the maximal height of the element of order $p$ in $G$, and we have $h_{G_{1}}\left(g_{1}\right)=h_{G}\left(g_{1}\right)$.

If the first sum does occur then $g_{1}=g_{1}^{(M)}+g_{1}^{(N)}$ where $g_{1}^{(M)}(\neq 0) \in \bar{M}$ and $g_{1}^{(N)} \in \bar{N}$.

Since $G=M \oplus N$, it follows that

$$
h_{G}\left(g_{1}\right)=\min \left(h_{M}\left(g_{1}^{(M)}\right), h_{N}\left(g_{1}^{(N)}\right)\right)=h-1
$$

which is the minimal height of the element of order $p$ in $G_{1}$, hence $h_{G}\left(g_{1}\right)=h_{G_{1}}\left(g_{1}\right)$.

Therefore, $\left\{g_{\alpha}\right\}$ is a pure independent subset of $G$. By [1, Lemma 11] the set $\left\{g_{\alpha}\right\}$ can be enlarged to a basis $\left\{g_{\beta}\right\}$ of $G$, which is the desired one.

Lemma V. A torsion free group $G$ has property (B) if and only if it is a free group of finite rank.

Proof. If $G$ is a free group of finite rank, by the theorem on the subgroups of a free group of finite rank, it has property (B).

It is well known that a group of order $\sigma$ is isomorphic to a factor group of a free group of $\operatorname{rank} \sigma$, and for any infinite cardinal $\rho$ there exists a group of order $\rho$ which is not a direct sum of cyclic groups. Since the group $G$ with property (B) is a free group, we shall show that it must be of finite rank. Suppose to the contrary that $G$ is of infinite rank. Then, select a subgroup $V$ of $G$ such that $G / V$ is not a direct sum of cyclic groups. It is clear that $G$ cannot have property (B) with respect to $V$.

THEOREM B. A group $G$ has property (B) if and only if either

(a) $G$ is a free group of finite rank, or

(b) $G$ is of the type 


$$
\sum_{p} \oplus\left(\sum_{\lambda_{p}} \oplus Z_{\lambda_{p}}\left(p^{h{ }^{p}}\right) \oplus \sum_{\mu_{p}} \oplus Z_{\mu_{p}}\left(p^{h_{p}+1}\right)\right) .
$$

Proof. The sufficiency follows from Lemma IV, Lemma V and the General lemma. Since (B) implies (A), $G$ must be a torsion-free group or a group of the type (b) by Theorem $A_{1}$. If $G$ is torsion-free, by Lemma V, $G$ must be a free group of finite rank. Therefore, the condition is also necessary.

4. Groups with property (C). We note first that (C) implies (A) and (B). It is immediate that (C) implies (A). Let $G$ have property (C) and let $\left\{h_{\alpha}\right\}$ be a maximal independent set in $G$. Then there exists another independent subset $\left\{g_{\alpha}\right\}$ of $G$ such that $h_{\alpha} \in\left(g_{\alpha}\right)$, and $\left(\left\{g_{\alpha}\right\}\right)$ is a direct summand of $G$. Since $\left\{h_{\alpha}\right\}$ is maximal, $G=\left(\left\{g_{\alpha}\right\}\right)$, that is $G$ is a direct sum of cyclic groups. If $\left\{h_{\alpha}\right\}$ is a basis of a subgroup $H$ of $G$, then the independent set $\left\{g_{\alpha}\right\}$ such that $h_{\alpha} \in\left(g_{\alpha}\right)$ is pure (since $\left(\left\{g_{\alpha}\right\}\right)$ is a direct summand) and can be enlarged to a basis of $G$ by $[\mathbf{1}$, Lemma 11]. Hence (C) implies (B).

LEMma VI. A primary group $G$ has property (C) if and only if it is of the type

$$
\sum_{\lambda} \oplus Z_{\lambda}\left(p^{h}\right)
$$

Proof. Since (C) implies (A), the group $G$ is of the type

$$
\sum_{\lambda} \oplus Z_{\lambda}\left(p^{h}\right) \oplus \sum_{\mu} \oplus Z_{\mu}\left(p^{h+1}\right) .
$$

If both sets of indices for $\lambda, \mu$ are non-empty, then there exists $\left(u_{1}\right) \oplus\left(u_{2}\right)$, a direct summand of $G$ such that $o\left(u_{1}\right)=h \geqq 1$ and $o\left(u_{2}\right)=h+1$. Now, consider the independent subset $\left\{p^{h-1} u_{1}, p^{h-1} u_{1}+p^{h} u_{2}\right\}$ of $G$. It is clear that

$$
p^{h} u_{2} \in\left(p^{h-1} u_{1}, p^{h-1} u_{1}+p^{h} u_{2}\right) .
$$

If there exists a direct summand $V=\left(\tilde{u}_{1}\right) \oplus\left(\tilde{u}_{2}\right)$ of $G$ such that $p^{h-1} u_{1} \in\left(\tilde{u}_{1}\right), p^{h-1} u_{1}+p^{h} u_{2} \in\left(\tilde{u}_{2}\right)$, then

$$
\left(p^{h-1} u_{1}\right)=\overline{\left(\tilde{u}_{1}\right)},\left(\mathrm{p}^{h-1} u_{1}+p^{h} u_{2}\right)=\overline{\left(\tilde{u}_{2}\right)} .
$$

Since $p^{h} u_{2} \in V$, we must have

$$
h_{V}\left(p^{h} u_{2}\right)=h=\min . \quad\left(h_{V}\left(p^{h-1} u_{1}\right), h_{V}\left(p^{h-1} u_{1}+p^{h} u_{2}\right)\right)=h-1,
$$

which is a contradiction, therefore the condition is necessary.

Conversely, if $G$ is of the type

$$
\sum_{\lambda} \oplus Z_{\lambda}\left(p^{h}\right),
$$


then it follows from Lemma III, that any element $h_{\alpha}$ of an independent subset $\left\{h_{\alpha}\right\}$ of $G$ can be contained in a cyclic direct summand of $G$, viz., $\left(u_{\alpha}\right)$. Now, we are going to prove that $\left\{u_{\alpha}\right\}$ is a pure independent subset of $G$, hence by [1, Theorem 7], $U=\sum_{\alpha} \oplus\left(u_{\alpha}\right)$ is a direct summand of $G$. The independence of $\left\{u_{\alpha}\right\}$ follows immediately from the independence of $\left\{h_{\alpha}\right\}$. Since every element $x$ of order $p$ in $U$ has height $h-1$ in $U$ which is the height of $x$ in $G, U$ is pure in $G$, by [1, Lemma 7]. Thus, $G$ has property (C).

LEMma VII. A torsion free group has property (C), if and only if $G=I$ (the integers).

Proof. If $G=I$, it clearly has property (C). Conversely, we shall prove that $G=I$ is also necessary. Suppose to the contrary that $G$ is a free group of rank not less than 2. (Since (C) implies (B), $G$ is a free group of finite rank.) Then, there exists a subgroup $V$ of $G$ such that

$$
G / V \cong Z(p) \oplus Z\left(p^{3}\right) \text {. }
$$

Let $u_{1}$ and $u_{2}$ be the generators of $Z(p)$ and $Z\left(p^{3}\right)$, respectively. Let $H$ be the complete inverse image of $\left(u_{1}+p u_{2}\right)$ with respect to the homomorphic mapping of $G$ onto $Z(p) \oplus Z\left(p^{3}\right)$. Then, $H$ is a free group of finite rank, therefore, a group with property (B). There exists a basis $\left\{v_{\alpha}\right\}$ of $V$ and a basis $\left\{h_{\beta}\right\}$ of $H$ such that for any $v_{\alpha}$ there is an $h_{\alpha} \in\left\{h_{\beta}\right\}$ with the property $v_{\alpha} \in\left(h_{\alpha}\right)$. If $G$ is a group with the property (C), then there exists a basis $\left\{g_{\gamma}\right\}$, such that $h_{\beta} \in\left(g_{\beta}\right)$ for all $\beta$, where $\beta_{\beta} \in\left\{g_{\gamma}\right\}$. Hence $G / V=\sum_{\alpha} \oplus\left(g_{\alpha}\right) /\left(v_{\alpha}\right)$ and $H / V=\sum_{\alpha} \oplus\left(h_{\alpha}\right) /\left(v_{\alpha}\right)$ where $\left(h_{i}\right) /\left(v_{\alpha}\right) \subset\left(g_{\alpha}\right) /\left(v_{\alpha}\right)$. Since $H / V \cong\left(u_{1}+p u_{2}\right)$ is cyclic, $H / V=\left(h_{\alpha_{1}}\right) /\left(v_{\alpha_{1}}\right)$ consists of a single summand of order $p^{2}$ which is contained in a cyclic direct summand of $G / V \cong Z(p) \oplus Z\left(p^{3}\right)$ of order $p^{3}$. Thus $u_{1}+p u_{2}=$ $p \bar{u}_{2}$, where $\left(\bar{u}_{2}\right)$ is a direct summand of $G / V$. This implies that $h_{G / V}\left(\bar{u}_{1}+p \bar{u}_{2}\right)=1$, but on the other hand it is clear that $h_{G / V}\left(u_{1}+p u_{2}\right)=0$. This contradiction completes the proof.

Theorem C. A group $G$ has property (C), if and only if either

(a) $G=I$, or

(b) $G$ is of the type

$$
\sum_{p} \oplus\left(\sum_{\lambda_{p}} \oplus Z_{\lambda_{p}}\left(p^{h} p\right)\right)
$$

Proof. Since (C) implies (A), the theorem follows from Theorem $\mathrm{A}_{1}$, Lemma VI, Lemma VII and the General lemma.

5. Conclusion. We have analyzed the groups characterized by 
their independent subsets. The classical theorem on the subgroups of a free group $U_{n}$ of finite rank is obviously a particular case of Theorem $\mathrm{B}$; Theorem A, Theorem $\mathrm{A}_{1}$ and Theorem $\mathrm{C}$ are its generalizations.

I extend my sincerest appreciation to Professor Ross. A. Beaumont for his suggestions, comments and corrections in the preparation of this paper. And I wish to thank my friends for their encouragement.

\section{REFERENCES}

1. I. Kaplansky, Infinite Abelian Groups, University of Michigan Publ. Math., No. 2, 1954.

2. A. G. Kurosh, Theory of Groups, I, 2nd edition. New York, 1955. (English translation)

TAIW AN UNIVERSITY 



\section{PACIFIC JOURNAL OF MATHEMATICS}

\section{EDITORS}

David Gilbarg

Stanford University

Stanford, California

R. A. Beaumont

University of Washington

Seattle 5, Washington

\author{
A. L. Whiteman
}

University of Southern California

Los Angeles 7, California

E. G. Straus

University of California

Los Angeles 24, California

\section{ASSOCIATE EDITORS}

\author{
E. F. BECKENBACH \\ C. E. BURGESS \\ M. HALL \\ E. HEWITT
}

\author{
A. HORN \\ V. GANAPATHY IYER \\ R. D. JAMES \\ M. S. KNEBELMAN
}

L. NACHBIN
I. NIVEN
T. G. OSTROM
H. L. ROYDEN

M. M. SCHIFFER

G. SZEKERES

F. WOLF

K. YOSIDA

\section{SUPPORTING INSTITUTIONS}

\author{
UNIVERSITY OF BRITISH COLUMBIA \\ CALIFORNIA INSTITUTE OF TECHNOLOGY \\ UNIVERSITY OF CALIFORNIA \\ MONTANA STATE UNIVERSITY \\ UNIVERSITY OF NEVADA \\ OREGON STATE COLLEGE \\ UNIVERSITY OF OREGON \\ UNIVERSITY OF SOUTHERN CALIFORNIA
}

\author{
STANFORD UNIVERSITY \\ UNIVERSITY OF UTAH \\ WASHINGTON STATE COLLEGE \\ UNIVERSITY OF WASHINGTON \\ AMERICAN MATHEMATICAL SOCIETY \\ CALIFORNIA RESEARCH CORPORATION \\ HUGHES AIRCRAFT COMPANY \\ THE RAMO-WOOLDRIDGE CORPORATION
}

Mathematical papers intended for publication in the Pacific Journal of Mathematics should be typewritten (double spaced), and the author should keep a complete copy. Manuscripts may be sent to any of the editors. All other communications to the editors should be addressed to the managing editor, E. G. Straus at the University of California, Los Angeles 24, California.

50 reprints per author of each article are furnished free of charge; additional copies may be obtained at cost in multiples of 50 .

The Pacific Journal of Mathematics is published quarterly, in March, June, September, and December. The price per volume (4 numbers) is $\$ 12.00$; single issues, $\$ 3.50$. Back numbers are available. Special price to individual faculty members of supporting institutions and to individual members of the American Mathematical Society: $\$ 4.00$ per volume; single issues, $\$ 1.25$.

Subscriptions, orders for back numbers, and changes of address should be sent to Pacific Journal of Mathematics, 2120 Oxford Street, Berkeley 4, California.

Printed at Kokusai Bunken Insatsusha (International Academic Printing Co., Ltd.), No. 6, 2-chome, Fujimi-cho, Chiyoda-ku, Tokyo, Japan.

\section{PUBLISHED BY PACIFIC JOURNAL OF MATHEMATICS, A NON-PROFIT CORPORATION}

The Supporting Institutions listed above contribute to the cost of publication of this Journal, but they are not owners or publishers and have no responsibility for its content or policies. 


\section{Pacific Journal of Mathematics}

\section{Vol. 8, No. 3 \\ May, 1958}

Michael Israel Aissen, A set function defined for convex plane domaines... . 383

Robert Ellis, Distal transformation groups ................... 401

Ciprian Foias, On a commutative extension of a commutative Banach algebra ....................................... 407

Jerry William Gaddum, Linear inequalities and quadratic forms ......... 411

Allen A. Goldstein and Elliott Ward Cheney, Jr., A finite algorithm for the solution of consistent linear equations and inequalities and for the Tchebycheff approximation of inconsistent linear equations...........

William L. Hart and T. S. Motzkin, Proof of the fundamental theorem on implicit functions by use of composite gradient corrections .......... 429

Henry Berge Helson, Conjugate series and a theorem of Paley .......... 437

Wu-Chung Hsiang, Abelian groups characterized by their independent subsets....................................... 447

John W. Lamperti, On the isometries of certain function-spaces ........ 459

Karel DeLeeuw and Walter Rudin, Extreme points and extremum problems

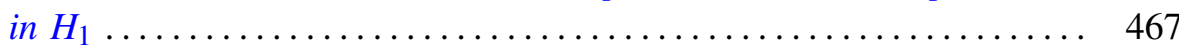

Eugene Lukacs, Some extensions of a theorem of Marcinkiewicz........ 487

George W. Mackey, Multiplicity free representations of finite groups ..... 503

Eben Matlis, Injective modules over Noetherian rings ............. 511

John William Neuberger, Continuous products and nonlinear integral equations

Lawrence Edward Payne and Hans F. Weinberger, New bounds for solutions of second order elliptic partial differential equations...

William T. Reid, A Prüfer transformation for differential systems ........ 575

Howard L. Rolf, The free lattice generated by a set of chains ...

K. M. Saksena, Inversion and representation theorems for a generalized

Laplace integral....................................... 597

Daniel Shanks, Two theorems of Gauss......................... 609

Paul Slepian, On the Lebesgue area of a doubled map ............... 613

Otto Szász and Nelson Paul Yeardley, Jr., The representation of an analytic function by general Laguerre series . ..................... 621

Alan C. Woods, On two-dimensional convex bodies ................. 635 